\title{
CHOI-DAVIS-JENSEN'S INEQUALITY AND GENERALIZED INVERSES OF LINEAR OPERATORS*
}

\author{
MAREK NIEZGODA ${ }^{\dagger}$
}

\begin{abstract}
In this paper, some extensions of recent results on Choi-Davis-Jensen's inequality due to Khosravi et al. [M. Khosravi, J.S. Aujla, S.S. Dragomir, and M.S. Moslehian. Refinements of ChoiDavis-Jensen's inequality. Bulletin of Mathematical Analysis and Applications, 3:127-133, 2011.] and Fujii et al. [J.-I. Fujii, J. Pečarić, and Y. Seo. The Jensen inequality in an external formula. Journal of Mathematical Inequalities, 6:473-480, 2012.] are presented. To this end, generalized inverses of positive linear maps are used.
\end{abstract}

Key words. Unital $C^{*}$-algebra, Operator convex/concave function, Positive linear map, ChoiDavis-Jensen's inequality, Generalized inverse.

AMS subject classifications. 15A09, 47A63, 26A51.

1. Introduction. As usual, the symbol $\mathbb{B}(H)$ stands for the $C^{*}$-algebra of all bounded linear operators on Hilbert space $H$ with inner product $\langle\cdot, \cdot\rangle$.

Throughout the paper, any $C^{*}$-algebra is assumed to be a closed $*$-subalgebra of $\mathbb{B}(H)$ for some Hilbert space $H$. In this situation, a self-adjoint operator $A$ in $C^{*}$ algebra $\mathcal{A}$ is said to be positive, written $0 \leq A$, if $\langle A x, x\rangle \geq 0$ for $x \in H$. If moreover $A$ is invertible, then $A$ is said to be strictly positive, written $0<A$. For self-adjoint elements $A$ and $B$ of a $C^{*}$-algebra $\mathcal{A}$, we write $A \leq B$ (resp., $A<B$ ) if $B-A$ is positive (resp., strictly positive) in $\mathcal{A}$. if

A continuous function $f: J \rightarrow \mathbb{R}$ on an interval $J \subset \mathbb{R}$ is called operator convex

$$
f(\alpha A+(1-\alpha) B) \leq \alpha f(A)+(1-\alpha) f(B)
$$

for $\alpha \in[0,1]$ and self-adjoint operators $A, B \in \mathcal{A}$ with spectra $\sigma(A), \sigma(B)$ in $J$. A continuous function $f: J \rightarrow \mathbb{R}$ on an interval $J \subset \mathbb{R}$ is called operator concave if $-f$ is operator convex.

A linear map $\Phi: \mathcal{A} \rightarrow \mathcal{B}$ between $C^{*}$-algebras $\mathcal{A}$ and $\mathcal{B}$ is said to be positive, if

$$
0 \leq A \text { implies } 0 \leq \Phi(A) \text { for } A \in \mathcal{A} \text {. }
$$

${ }^{*}$ Received by the editors on October 12, 2012. Accepted for publication on June 25, 2013. Handling Editor: Moshe Goldberg.

${ }^{\dagger}$ Department of Applied Mathematics and Computer Science, University of Life Sciences in Lublin, Akademicka 13, 20-950 Lublin, Poland (marek.niezgoda@up.lublin.pl, bniezgoda@wp.pl). 
If (1.1) is replaced by the following condition

$$
0<A \text { implies } 0<\Phi(A) \text { for } A \in \mathcal{A} \text {, }
$$

then $\Phi$ is said to be strictly positive.

A linear map $\Phi: \mathcal{A} \rightarrow \mathcal{B}$ between unital $C^{*}$-algebras $\mathcal{A}$ and $\mathcal{B}$ is called unital if $\Phi(I)=I$, where $I$ denotes the unities of the algebras.

The Choi-Davis-Jensen (C-D-J) inequality asserts that if $f$ is an operator convex function on an interval $J$, and $\Phi$ is a unital positive linear map from a unital $C^{*}$ algebra $\mathcal{A}$ into a unital $C^{*}$-algebra $\mathcal{B} \subset \mathbb{B}(K)$ with a Hilbert space $K$, then

$$
f(\Phi(A)) \leq \Phi(f(A))
$$

for every self-adjoint operator $A \in \mathcal{A}$ with spectrum contained in $J$ [7, p. 128].

Furthermore, if $f$ is an operator convex function on an interval $J$, and $\Phi_{1}, \ldots, \Phi_{n}$ are positive linear maps from a unital $C^{*}$-algebra $\mathcal{A}$ into a unital $C^{*}$-algebra $\mathcal{B}$ such that $\sum_{i=1}^{n} \Phi_{i}(I)=I$, then

$$
f\left(\sum_{i=1}^{n} \Phi_{i}\left(A_{i}\right)\right) \leq \sum_{i=1}^{n} \Phi_{i}\left(f\left(A_{i}\right)\right)
$$

for every self-adjoint operators $A_{i} \in \mathcal{A}, i=1,2, \ldots, n$, with spectra contained in $J$ [5, pp. 63-64].

Here we cite some recent results due to Khosravi et al. [7, Theorem 2.2] and Fujii et al. 4, Theorem 3.1]. For related results, see [1, 8, 9].

Theorem A. (Khosravi et al. [7, Theorem 2.2]) Let $\Phi_{1}, \ldots, \Phi_{n}$ be strictly positive linear maps from a unital $C^{*}$-algebra $\mathcal{A}$ into a unital $C^{*}$-algebra $\mathcal{B}$ and let $\Phi=\sum_{i=1}^{n} \Phi_{i}$ be unital.

If $f$ is an operator convex function on an interval $J$, then

(1.4) $f(\Phi(A)) \leq \sum_{i=1}^{n} \Phi_{i}(I)^{1 / 2} f\left(\Phi_{i}(I)^{-1 / 2} \Phi_{i}(A) \Phi_{i}(I)^{-1 / 2}\right) \Phi_{i}(I)^{1 / 2} \leq \Phi(f(A))$

for every self-adjoint operator $A \in \mathcal{A}$ with spectrum contained in $J$.

For Hilbert spaces $H$ and $K$, the symbol $P[\mathbb{B}(H), \mathbb{B}(K)]$ stands for the convex cone of all positive linear maps between $\mathbb{B}(H)$ and $\mathbb{B}(K)$.

Theorem B. (Fujii et al. [4, Theorem 3.1]) Let $f: J \rightarrow \mathbb{R}$ be a continuous function on an interval $J$. Then $f$ is an operator concave if and only if

$$
f(\Phi(A)-\Psi(B)) \leq \Phi(I)^{1 / 2} f\left(\Phi(I)^{-1 / 2} \Phi(A) \Phi(I)^{-1 / 2}\right) \Phi(I)^{1 / 2}-\Psi f(B)
$$


for all $\Phi, \Psi \in P[\mathbb{B}(H), \mathbb{B}(K)]$ such that $\Phi(I)-\Psi(I)=I$ and $\Phi(I)>0$, and for all self-adjoint operators $A$ and $B$ with spectra $\sigma(A), \sigma(B), \sigma(\Phi(A)-\Psi(B)) \subset J$.

The purpose of the present paper is to extend the above theorems. For an operator convex function $f$, we demonstrate a refinement of the Choi-Davis-Jensen inequality involving the transformation $T f T^{-}$, where $T^{-}$denotes a reflexive generalized inverse of a positive linear map $T$. We also provide several corollaries describing some special cases. Such an approach allows to obtain some complementary statements to Theorem A. On the other hand, in order to generalize Theorem B, we show a C-D-J type inequality with the help of generalized inverses of some auxiliary operators. Finally, we discuss some variants of such a result.

2. Refining Choi-Davis-Jensen's inequality. A generalized inverse of a linear map $T: \mathcal{A} \rightarrow \mathcal{B}$ between linear spaces is a linear map $T^{-}: \mathcal{B} \rightarrow \mathcal{A}$ satisfying $T T^{-} T=T$. If in addition $T^{-} T T^{-}=T^{-}$then $T^{-}$is called a reflexive generalized inverse of $T$.

For instance, it is known that if $T \in \mathbb{B}(H)$ with a Hilbert space $H$, then there exists a generalized inverse of $T$ if and only if $T$ has closed range [6].

Throughout the paper, whenever the symbol $T^{-}$is used, it is assumed that there exists a generalized inverse $T^{-}$of a linear map $T$.

In what follows, we shall use positive reflexive generalized inverse $T^{-}$which means that $T^{-}: \mathcal{B} \rightarrow \mathcal{A}$ is positive and reflexive, provided that $\mathcal{A}$ and $\mathcal{B}$ are $C^{*}$-algebras. If a positive linear map $T: \mathcal{A} \rightarrow \mathcal{B}$ is invertible then $T$ is called invertible positive linear map. By $\operatorname{Ran}(T)$ we denote the range of a linear map $T$.

In the sequel, in the interest of simplicity, we often write $f \Theta(C)$ and $\Theta f(C)$ in place of $f(\Theta(C))$ and $\Theta(f(C))$, respectively, for a function $f$, a linear map $\Theta$ and an element $C$ in a $C^{*}$-algebra $\mathcal{A}$. For example, $\Theta(C)^{p}$ means $(\Theta(C))^{p}$ for exponent $p$.

ThEOREM 2.1. Let $\Phi_{1}, \ldots, \Phi_{n}$ be positive linear maps from a unital $C^{*}$-algebra $\mathcal{A}$ into a unital $C^{*}$-algebra $\mathcal{B}$ and let $\Phi=\sum_{i=1}^{n} \Phi_{i}$ be unital. For $i=1,2, \ldots, n$, let $T_{i}: \mathcal{A} \rightarrow \mathcal{B}$ be a positive linear map with a positive reflexive generalized inverse $T_{i}^{-}$ satisfying

$$
\begin{array}{r}
\Phi_{i}(I)=T_{i}(I), \\
\operatorname{Ran}\left(\Phi_{i}\right) \subset \operatorname{Ran}\left(T_{i}\right), \\
I \in \operatorname{Ran}\left(T_{i}^{-}\right) .
\end{array}
$$


If $f$ is an operator convex function on an interval $J$, then

$$
f(\Phi(A)) \leq \sum_{i=1}^{n} T_{i} f T_{i}^{-} \Phi_{i}(A) \leq \Phi(f(A))
$$

for every self-adjoint operator $A \in \mathcal{A}$ with spectrum contained in $J$.

Proof. (Based on the idea of the proof of [7, Theorem 2.2].)

Fix arbitrarily a self-adjoint element $A \in \mathcal{A}$ with spectrum contained in $J$.

By (2.2), we get $T_{i} T_{i}^{-} \Phi_{i}(A)=\Phi_{i}(A)$ for $i=1,2, \ldots, n$. Moreover, $\sum_{i=1}^{n} T_{i}(I)=$ $\sum_{i=1}^{n} \Phi_{i}(I)=I$ by (2.1). So, in light of inequality (1.3), we deduce that

$$
f(\Phi(A))=f\left(\sum_{i=1}^{n} \Phi_{i}(A)\right)=f\left(\sum_{i=1}^{n} T_{i} T_{i}^{-} \Phi_{i}(A)\right) \leq \sum_{i=1}^{n} T_{i} f T_{i}^{-} \Phi_{i}(A) .
$$

Thus, the first inequality in (2.4) is proven.

To see the second inequality in (2.4), observe from (2.1) and (2.3) that $T_{i}^{-} \Phi_{i}(I)=$ $I$ for $i=1,2, \ldots, n$. Furthermore, $T_{i}^{-} \Phi_{i}$ is positive, because $\Phi_{i}$ and $T_{i}^{-}$are so. Thus, $T_{i}^{-} \Phi_{i}$ is a unital positive map. Now, by (1.2) we infer that

$$
f T_{i}^{-} \Phi_{i}(A) \leq T_{i}^{-} \Phi_{i} f(A) \text { for } i=1,2, \ldots, n .
$$

Hence, by (2.2),

$$
T_{i} f T_{i}^{-} \Phi_{i}(A) \leq T_{i} T_{i}^{-} \Phi_{i} f(A)=\Phi_{i} f(A) \text { for } i=1,2, \ldots, n,
$$

because $T_{i}$ is positive. Therefore, we obtain

$$
\sum_{i=1}^{n} T_{i} f T_{i}^{-} \Phi_{i}(A) \leq \sum_{i=1}^{n} \Phi_{i} f(A)=\Phi(f(A)),
$$

which completes the proof. $\square$

A special case of Theorem 2.1 is for $\Phi_{i}=T_{i} S_{i}$ with a unital positive map $S_{i}$ : $\mathcal{A} \rightarrow \mathcal{A}, i=1,2, \ldots, n$. Then conditions (2.1)-(2.2) hold automatically and therefore can be deleted.

Corollary 2.2. Let $T_{1}, \ldots, T_{n}$ be positive linear maps from a unital $C^{*}$-algebra $\mathcal{A}$ into a unital $C^{*}$-algebra $\mathcal{B}$ such that $\sum_{i=1}^{n} T_{i}$ is unital. Let $S_{i}: \mathcal{A} \rightarrow \mathcal{A}$ be a unital positive linear map, $i=1,2, \ldots, n$. Assume $T_{i}^{-}$is a positive reflexive generalized 
inverse of $T_{i}$ such that $I \in \operatorname{Ran}\left(T_{i}^{-}\right), i=1,2, \ldots, n$. If $f$ is an operator convex function on an interval $J$, then

$$
f\left(\sum_{i=1}^{n} T_{i} S_{i}(A)\right) \leq \sum_{i=1}^{n} T_{i} f T_{i}^{-} T_{i} S_{i}(A) \leq \sum_{i=1}^{n} T_{i} S_{i} f(A)
$$

for every self-adjoint operator $A \in \mathcal{A}$ with spectrum contained in $J$.

Condition (2.2) can be removed from Theorem 2.1 if $T_{i}$ is of "full column rank", i.e., $\operatorname{Ran}\left(T_{i}\right)=\mathcal{B}$. Likewise, condition (2.3) can be removed if $T_{i}^{-}$is of "full column rank", i.e., $\operatorname{Ran}\left(T_{i}^{-}\right)=\mathcal{A}$. For instance, if $T_{i}$ is invertible, then Theorem 2.1 gives the folowing.

Corollary 2.3. Let $\Phi_{1}, \ldots, \Phi_{n}$ be positive linear maps from a unital $C^{*}$-algebra $\mathcal{A}$ into a unital $C^{*}$-algebra $\mathcal{B}$ and let $\Phi=\sum_{i=1}^{n} \Phi_{i}$ be unital. Let $T_{i}: \mathcal{A} \rightarrow \mathcal{B}$ be an invertible positive linear map such that the inverse $T_{i}^{-1}$ is positive and $T_{i}(I)=\Phi_{i}(I)$, $i=1,2, \ldots, n$. If $f$ is an operator convex function on an interval $J$, then

$$
f(\Phi(A)) \leq \sum_{i=1}^{n} T_{i} f T_{i}^{-1} \Phi_{i}(A) \leq \Phi(f(A))
$$

for every self-adjoint operator $A \in \mathcal{A}$ with spectrum contained in $J$.

It is readily seen that Theorem A in Section 1 can be recovered from Corollary 2.3 applied to the maps

$$
T_{i}:=\Phi_{i}(I)^{1 / 2}(\cdot) \Phi_{i}(I)^{1 / 2} \text { and } T_{i}^{-1}:=\Phi_{i}(I)^{-1 / 2}(\cdot) \Phi_{i}(I)^{-1 / 2}
$$

for strictly positive linear maps $\Phi_{i}, i=1,2, \ldots, n$.

With the additional assumption that the maps $T_{1}, \ldots, T_{n}$ are invertible, Corollary 2.2 yields next result as follows.

COROllary 2.4. Let $T_{1}, \ldots, T_{n}$ be invertible positive linear maps from a unital $C^{*}$-algebra $\mathcal{A}$ into a unital $C^{*}$-algebra $\mathcal{B}$ such that $\sum_{i=1}^{n} T_{i}$ is unital. Let $S_{i}: \mathcal{A} \rightarrow \mathcal{A}$ be a unital positive linear map, $i=1,2, \ldots, n$. Assume $T_{i}^{-1}$ is positive. If $f$ is an operator convex function on an interval $J$, then

$$
f\left(\sum_{i=1}^{n} T_{i} S_{i}(A)\right) \leq \sum_{i=1}^{n} T_{i} f S_{i}(A) \leq \sum_{i=1}^{n} T_{i} S_{i} f(A)
$$

for every self-adjoint operator $A \in \mathcal{A}$ with spectrum contained in $J$.

It is well known that the power functions

$$
f(t)=t^{p} \quad \text { on } J=[0, \infty), p \in[1,2], \quad \text { and } \quad f(t)=t^{-1} \quad \text { on } J=(0, \infty)
$$


are operator convex functions, while

$$
f(t)=t^{p} \quad \text { on } J=[0, \infty), p \in(0,1],
$$

are operator concave functions on $J=(0, \infty)$ (see e.g. [7, pp. 130-131]).

As a consequence of Corollary 2.3 we obtain the following.

Corollary 2.5. Let $\Phi_{1}, \ldots, \Phi_{n}$ be positive linear maps from a unital $C^{*}$-algebra $\mathcal{A}$ into a unital $C^{*}$-algebra $\mathcal{B}$ and let $\Phi=\sum_{i=1}^{n} \Phi_{i}$ be unital. Let $T_{i}: \mathcal{A} \rightarrow \mathcal{B}$ be an invertible positive linear map such that the inverse $T_{i}^{-1}$ is positive and $T_{i}(I)=\Phi_{i}(I)$, $i=1,2, \ldots, n$. Then the following hold:

(i) For $p \in[1,2]$,

$$
\Phi(A)^{p} \leq \sum_{i=1}^{n} T_{i}\left(T_{i}^{-1} \Phi_{i}(A)\right)^{p} \leq \Phi\left(A^{p}\right)
$$

for every positive operator $A \in \mathcal{A}$,

(ii) For $p=-1$,

$$
\Phi(A)^{-1} \leq \sum_{i=1}^{n} T_{i}\left(T_{i}^{-1} \Phi_{i}(A)\right)^{-1} \leq \Phi\left(A^{-1}\right)
$$

for every strictly positive operator $A \in \mathcal{A}$.

For $p \in(0,1]$, the inequalities (2.10) will be reversed.

We now show a method to refine inequalities of (2.4) in Theorem 2.1.

TheOREM 2.6. Let $\Phi_{1}, \ldots, \Phi_{n}$ be positive linear maps from a unital $C^{*}$-algebra $\mathcal{A}$ into a unital $C^{*}$-algebra $\mathcal{B}$ and let $\Phi=\sum_{i=1}^{n} \Phi_{i}$ be unital. For $i=1,2, \ldots, n$, let $T_{i}: \mathcal{A} \rightarrow \mathcal{B}$ and $R_{i}: \mathcal{A} \rightarrow \mathcal{B}$ be positive linear maps with positive reflexive generalized inverses $T_{i}^{-}$and $R_{i}^{-}$, respectively, such that

$$
\begin{array}{r}
\Phi_{i}(I)=R_{i}(I)=T_{i}(I), \\
\operatorname{Ran}\left(\Phi_{i}\right) \subset \operatorname{Ran}\left(T_{i}\right) \subset \operatorname{Ran}\left(R_{i}\right), \\
I \in \operatorname{Ran}\left(T_{i}^{-}\right) \cap \operatorname{Ran}\left(R_{i}^{-}\right) .
\end{array}
$$

If $f$ is an operator convex function on an interval $J$, then

$$
f(\Phi(A)) \leq \sum_{i=1}^{n} R_{i} f R_{i}^{-} \Phi_{i}(A) \leq \sum_{i=1}^{n} T_{i} f T_{i}^{-} \Phi_{i}(A) \leq \Phi(f(A))
$$

for every self-adjoint operator $A \in \mathcal{A}$ with spectrum contained in $J$. 
Proof. Assume that $A \in \mathcal{A}$ is self-adjoint with spectrum contained in $J$.

It follows that $R_{i}^{-} T_{i}(I)=I$ for $i=1,2, \ldots, n$, because $T_{i}(I)=R_{i}(I)$ and $I \in$ $\operatorname{Ran}\left(R_{i}^{-}\right)$. Additionally, $R_{i}^{-} T_{i}$ is positive, since $T_{i}$ and $R_{i}^{-}$are so. Thus, $R_{i}^{-} T_{i}$ is a unital positive linear map.

Moreover, $T_{i} T_{i}^{-} \Phi_{i}(A)=\Phi_{i}(A)$ by (2.13). According to (1.2), we conclude that

$$
f R_{i}^{-} \Phi_{i}(A)=f R_{i}^{-} T_{i} T_{i}^{-} \Phi_{i}(A) \leq R_{i}^{-} T_{i} f T_{i}^{-} \Phi_{i}(A)
$$

Hence, via the positivity of $R_{i}$ and (2.13), we get

$$
R_{i} f R_{i}^{-} \Phi_{i}(A) \leq R_{i} R_{i}^{-} T_{i} f T_{i}^{-} \Phi_{i}(A)=T_{i} f T_{i}^{-} \Phi_{i}(A), \quad i=1,2, \ldots, n,
$$

and further

$$
\sum_{i=1}^{n} R_{i} f R_{i}^{-} \Phi_{i}(A) \leq \sum_{i=1}^{n} T_{i} f T_{i}^{-} \Phi_{i}(A)
$$

To complete the proof it is now sufficient to apply Theorem 2.1 .

3. Further refinements of Choi-Davis-Jensen's inequality. In this section, we aim to give an extension of Theorem B (see Section 1). We prefer to state it in version for operator convex functions.

From now on we assume that $\mathcal{A}=\mathbb{B}(H)$ and $\mathcal{B}=\mathbb{B}(K)$ are unital $C^{*}$-algebras of all bounded linear operators on some Hilbert spaces $H=K$, respectively. As previously, we denote by $P[\mathbb{B}(H), \mathbb{B}(K)]$ the set of all positive linear maps from $\mathbb{B}(H)$ to $\mathbb{B}(K)$.

The forthcoming lemma extends a version of Theorem 2.1 for $n=1$.

Lemma 3.1. Let $f: J \rightarrow \mathbb{R}$ be an operator convex function on an interval $J$.

Then the following inequality holds:

$$
f \Phi(A) \leq T f T^{-} \Phi(A) \leq T T^{-} f \Phi(A) \leq \Phi f(A)
$$

for all self-adjoint operators $A \in \mathbb{B}(H)$ with spectrum $\sigma(A)$ contained in $J$, and for all unital positive operators $\Phi, T \in P[\mathbb{B}(H), \mathbb{B}(K)]$ with a positive reflexive generalized inverse $T^{-}$such that

$$
\begin{array}{r}
\operatorname{Ran}(\Phi) \subset \operatorname{Ran}(T), \\
I \in \operatorname{Ran}\left(T^{-}\right) .
\end{array}
$$


Proof. Fix arbitrarily a self-adjoint operator $A \in \mathbb{B}(H)$ with spectrum $\sigma(A) \subset J$.

From (3.3) we get $T^{-} T(I)=I$. But $T$ is unital. For this reason, $T^{-}(I)=I$. Consequently, $T^{-}$is a unital positive linear map. In addition, $\Phi$ and $T$ are so. Because $f$ is operator convex, we find from C-D-J inequality (1.2) that

$$
f T T^{-} \Phi(A) \leq T f T^{-} \Phi(A) \leq T T^{-} f \Phi(A) \leq T T^{-} \Phi f(A) .
$$

Furthermore, by (3.2), we can write

$$
T T^{-} \Phi(A)=\Phi(A) \text { and } T T^{-} \Phi f(A)=\Phi f(A) .
$$

This and (3.4) give (3.1), as wanted. This finishes the proof. $\square$

In general, the middle inequality in (3.1) refines the Choi-Davis-Jensen inequality (1.2) under the assumptions (3.2)-(3.3) with unital positive maps $\Phi$ and $T$. However, if moreover $T$ is invertible then (3.1) becomes the classical C-D-J inequality.

In the next theorem, we generalize the middle inequality of (3.1) by introducing an additional positive linear operator $\Psi$. Thus, we extend the C-D-J inequality due to Fujii et al. (see [4, Theorem 3.1]).

ThEOREM 3.2. Let $f: J \rightarrow \mathbb{R}$ be a continuous function on an interval $J$. The following two statements are equivalent:

(i) $f$ is an operator convex function on an interval $J$.

(ii) The following inequality holds:

$$
T f T^{-} \Phi(A)-\Psi f(B) \leq T T^{-} f(\Phi(A)-\Psi(B))
$$

for all self-adjoint operators $A, B \in \mathbb{B}(H)$ with spectra $\sigma(A), \sigma(B), \sigma(\Phi(A)$ $\Psi(B)$ ) contained in $J$, and for all $\Phi, \Psi, T \in P[\mathbb{B}(H), \mathbb{B}(K)]$ satisfying $\Phi(I)-$ $\Psi(I)=I$ with a positive reflexive generalized inverse $T^{-}$such that

$$
\begin{array}{r}
\Phi(I)=T(I), \\
\operatorname{Ran}(\Psi) \subset \operatorname{Ran}(T), \\
I \in \operatorname{Ran}\left(T^{-}\right) .
\end{array}
$$

Proof. (i) $\Rightarrow$ (ii). By (3.6) we have $T(I)=\Psi(I)+I$ and next $I=T^{-} \Psi(I)+T^{-}(I)$ by (3.8). Moreover, $T^{-} \Psi$ and $T^{-}$are positive maps.

Because $f$ is operator convex, we derive

$$
f\left(T^{-} \Psi(C)+T^{-}(D)\right) \leq T^{-} \Psi f(C)+T^{-} f(D)
$$

for self-adjoint operators $C \in \mathbb{B}(H)$ and $D \in \mathbb{B}(K)$ with spectra contained in $J$. 
Substituting $C:=B$ and $D:=\Phi(A)-\Psi(B)$ yields

$$
f T^{-} \Phi(A)=f\left(T^{-} \Psi(B)+T^{-}(\Phi(A)-\Psi(B))\right) \leq T^{-} \Psi f(B)+T^{-} f(\Phi(A)-\Psi(B)) .
$$

Hence, by the positivity of $T$ and (3.7), we deduce that

$$
T f T^{-} \Phi(A) \leq \Psi f(B)+T T^{-} f(\Phi(A)-\Psi(B)) .
$$

This implies (3.5), as required.

(ii) $\Rightarrow$ (i). Take any strictly positive map $\Phi \in P[\mathbb{B}(H), \mathbb{B}(K)]$. Then $\Phi(I)>0$ [7. p. 127]. Put $T=\Phi(I)^{1 / 2}(\cdot) \Phi(I)^{1 / 2}$. Then $T$ is invertible and $T^{-}=T^{-1}=$ $\Phi(I)^{-1 / 2}(\cdot) \Phi(I)^{-1 / 2}$. Moreover, conditions (3.6), (3.7) and (3.8) are satisfied.

For these reasons, by (3.5) we establish the inequality

$$
\Phi(I)^{1 / 2}\left(f\left(\Phi(I)^{-1 / 2} \Phi(A) \Phi(I)^{-1 / 2}\right)\right) \Phi(I)^{1 / 2}-\Psi f(B) \leq f(\Phi(A)-\Psi(B))
$$

for all self-adjoint operators $A, B \in \mathbb{B}(H)$ with spectra $\sigma(A), \sigma(B), \sigma(\Phi(A)-\Psi(B))$ contained in $J$, and for all $\Phi, \Psi \in P[\mathbb{B}(H), \mathbb{B}(K)]$ satisfying $\Phi(I)-\Psi(I)=I$ and $\Phi(I)>0$.

Finally, by [4, Theorem 3.1] we infer that $f$ is operator convex (see Theorem B in Section 1).

It is interesting that if $T=\Phi$ then 3.5 gives

$$
\Phi f(A)-\Psi f(B) \leq \Phi \Phi^{-} f(\Phi(A)-\Psi(B)) \text { for } A \in \operatorname{Ran}\left(\Phi^{-}\right) \text {and } B \in \mathcal{A}
$$

with spectra $\sigma(A), \sigma(B), \sigma(\Phi(A)-\Psi(B)) \subset J$.

If in addition $f(\Phi(A)-\Psi(B)) \in \operatorname{Ran}(\Phi)$ then $\Phi \Phi^{-}$can be removed from (3.9). For example, if $\Phi$ is invertible then the operator $\Phi \Phi^{-}$is the identity, and the restriction $A \in \operatorname{Ran}\left(\Phi^{-}\right)$can be replaced by $A \in \mathcal{A}$.

In the last theorem, whenever $T$ is "of full column rank", i.e., $\operatorname{Ran} T=\mathcal{B}$, then condition (3.7) holds automatically and therefore can be deleted. Likewise, condition (3.8) can be deleted, if $T^{-}$is "of full column rank", i.e., $\operatorname{Ran} T^{-}=\mathcal{A}$. Thus, we obtain the following result for invertible $T$.

Corollary 3.3. Let $f: J \rightarrow \mathbb{R}$ be a continuous function on an interval $J$. The following two statements are equivalent:

(i) $f$ is an operator convex function on an interval $J$.

(ii) The following inequality holds:

$$
T f T^{-1} \Phi(A)-\Psi f(B) \leq f(\Phi(A)-\Psi(B))
$$


for all self-adjoint operators $A, B \in \mathcal{A}$ with spectra $\sigma(A), \sigma(B), \sigma(\Phi(A)-$ $\Psi(B)$ ) contained in $J$, and for all $\Phi, \Psi, T \in P(\mathbb{B}(H), \mathbb{B}(K))$ satisfying $\Phi(I)$ $\Psi(I)=I$ with invertible $T$ and positive inverse $T^{-1}$ such that $\Phi(I)=T(I)$.

It is not hard to verify that the mentioned result of Fujii et al. 4, Theorem 3.1] (see Theorem B in Section 11) can be recovered from (3.10) with the substitution

$$
T:=\Phi(I)^{1 / 2}(\cdot) \Phi(I)^{1 / 2} \text { and } T^{-1}:=\Phi(I)^{-1 / 2}(\cdot) \Phi(I)^{-1 / 2}
$$

for strictly positive linear maps $\Phi$.

By virtue of (3.10), for $T:=\Phi$ with invertible $\Phi$, we get

$$
\Phi f(A)-\Psi f(B) \leq f(\Phi(A)-\Psi(B))
$$

(cf. [4, Remark 3.2]).

By making use of Corollary 3.3 applied to $f(t)=t^{p}, p \in[1,2], J=[0, \infty)$ (see $(2.8)$ ), we obtain

COROLlary 3.4. The following inequality holds:

$$
T\left(T^{-1} \Phi(A)\right)^{p}-\Psi\left(B^{p}\right) \leq(\Phi(A)-\Psi(B))^{p}, \quad p \in[1,2],
$$

for all positive operators $A, B \in \mathcal{A}$ with $\sigma(\Phi(A)-\Psi(B)) \subset[0, \infty)$, for all $\Phi, \Psi, T \in$ $P[\mathbb{B}(H), \mathbb{B}(K)]$ satisfying $\Phi(I)-\Psi(I)=I$ and $\Phi(I)=T(I)$ with invertible $T$ and positive inverse $T^{-1}$.

Acknowledgment. The author wishes to thank an anonymous referee for useful comments improving the previous version of the manuscript.

\section{REFERENCES}

[1] J. Antezana, P. Massey, and D. Stojanoff. Jensen's inequality for spectral order and submajorization. Journal of Mathematical Analysis and Applications, 331:297-307, 2007.

[2] M.D. Choi. A Schwarz inequality for positive linear maps on $C^{*}$-algebras. Illinois Journal of Mathematics, 18:565-574, 1974.

[3] C. Davis. A Schwarz inequality for convex operator functions. Proceedings of the American Mathematical Society, 8:42-44, 1957.

[4] J.-I. Fujii, J. Pečarić, and Y. Seo. The Jensen inequality in an external formula. Journal of Mathematical Inequalities, 6:473-480, 2012.

[5] F. Hansen, J. Pečarić, and I. Perić. Jensen's operator inequality and its converses. Mathematica Scandinavica, 100:61-73, 2007.

[6] R. Harte and M. Mbekhta. On generalized inverses in $C^{*}$-algebras. Studia Mathematica, 103:71$77,1992$.

[7] M. Khosravi, J.S. Aujla, S.S. Dragomir, and M.S. Moslehian. Refinements of Choi-DavisJensen's inequality, Bulletin of Mathematical Analysis and Applications, 3:127-133, 2011. 
[8] J. Mićić, Z. Pavić, and J. Pečarić. Jensen's inequality for operators without operator convexity. Linear Algebra and its Applications, 434:1228-1237, 2011.

[9] J. Mićić, J. Pečarić, and J. Perić. Refined Jensen's operator inequality with condition on spectra. Operators and Matrices, 7:293-308, 2013.

[10] C.R. Rao and S.M. Mitra. Generalized inverse of a matrix and its applications. Proceedings of the Sixth Berkeley Symposium on Mathematical Statistics and Probability, University of California Press, 1:601-620, 1972. 